\title{
Étude sur le patrimoine phoenicicole de Kidal au nord du Mali
}

\author{
Souad BABAHANI ${ }^{1 *}$, Aissa TOGO ${ }^{1}$, Slimane HANNACHI ${ }^{2}$
}

${ }^{1}$ Lab. Bio-Ressour. Sahar., Univ. Ouargla, Ouargla, Algérie,

bbhsouad@gmail.com

${ }^{2}$ Commis. Dév. Agric. Rég. Sahar., Ouargla, Algérie
* Correspondance et tirés à part

Reçu le 12 avril 2011 Accepté le 20 juin 2011

Fruits, 2012, vol. 67, p. 77-86 (C) 2012 Cirad/EDP Sciences All rights reserved DOI: $10.1051 /$ fruits/2011071 www.fruits-journal.org

RESUMEN ESPAÑOL, p. 86

\section{Study on the date palm heritage of Kidal, in the North of Mali.}

Abstract - Introduction. The populations of Kidal, in the North of Mali, traditionally nomadic, are directing themselves gradually towards farming because of recurring droughts; date palm culture, although well represented, is still not very developed in this area. Our study is within the scope of the development projects of date palm in the North of Mali. It aimed to take an inventory of and characterize the phytogenetic resources of the date palm in order to draw up strategies for the development of this heritage. Materials and methods. Our study was carried out, in the area of Kidal, in nine main locations known for a long time for growing date palm. We determined the full number of plants observed in the area, the ratio of the productive plants, the sex distribution, the cultivars represented, the plant ages and their ripening times. Characterization of the major cultivars was determined based on vegetative characters and the morphological characteristics of inflorescences, fruits and seeds. Results and discussion. We listed more than 4000 date palms in the sampled area; the proportions of male palm trees appeared high. The location of Tessalit seems to be the most significant and oldest palm plantation of the area studied. It contains 39\% of the effective total. Our study made it possible to identify 18 cultivars. The multiple correspondence factorial analysis for characterization of the cultivars in the Kidal area showed that inter-cultivar variability seemed to be very high whatever the studied characters; on the other hand, intra-cultivar variability was low. Proximities were defined between the local Tigaza cultivars and the Algerian Tigaza.

Mali / Pboenix dactylifera / genetic resources / surveys / stand characteristics / genetic variation

\section{Étude du patrimoine phoenicicole de Kidal au nord du Mali.}

Résumé - Introduction. Les populations de Kidal, au nord du Mali, traditionnellement nomades, s'orientent progressivement vers l'agriculture du fait de sécheresses récurrentes ; la culture du palmier dattier bien que représentée est encore peu élaborée dans cette région. Notre étude s'est inscrite dans le cadre des projets de développement de la phoeniciculture au nord du Mali. Elle a eu pour objectif de recenser et de caractériser les ressources phytogénétiques du palmier dattier afin d'envisager des stratégies de développement de ce patrimoine. Matériel et méthodes. Notre étude a été réalisée, dans la région de Kidal, dans neuf sites principaux connus depuis longtemps pour la culture du palmier dattier. Nous avons déterminé le nombre total de pieds observés dans la région, l'effectif des pieds productifs, la répartition des sexes, les cultivars représentés, l'âge des pieds et leurs périodes de maturation. Une caractérisation des principaux cultivars a été établie en se basant sur des caractères végétatifs et des caractères morphologiques des inflorescences, des fruits et des graines. Résultats et discussion. Nous avons recensé plus de 4000 palmiers dattiers dans la région échantillonnée ; les proportions de palmiers mâles se sont révélées élevées. Le site de Tessalit semble héberger la palmeraie la plus importante et la plus ancienne. Il présente $39 \%$ de l'effectif total des palmiers dattiers recensés. Notre étude a permis d'identifier 18 cultivars. Les analyses factorielles des correspondances multiples sur la caractérisation des cultivars de la région de Kidal ont montré que la variabilité inter-cultivars semble être très importante quels que soient les caractères étudiés ; en revanche, la variabilité intra-cultivar est faible. Des proximités ont été définies entre les cultivars de Tigazas locaux et la Tigaza algérienne.

Mali / Phoenix dactylifera / ressource génétique / enquête / caractéristique du peuplement / variation génétique 


\section{S. Babahani et al.}

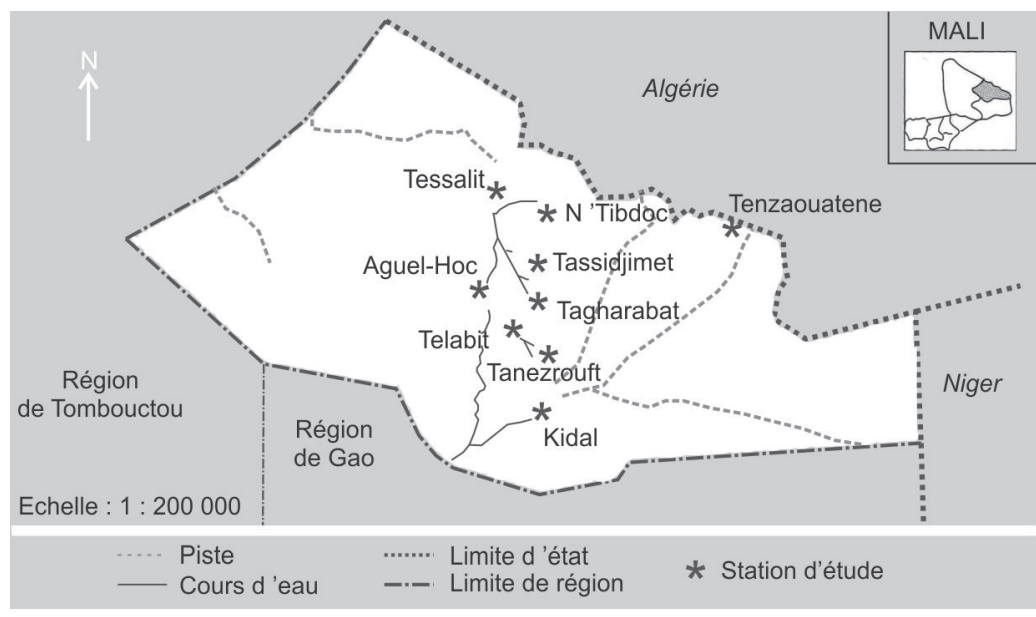

Figure 1.

Situation géographique de Kidal au nord-est du Mali et sites visités lors d'une prospection destinée à évaluer le patrimoine phoenicicole de cette région [2]. zone marginale pour la culture du dattier,

\section{Introduction}

Le Mali est un pays de l'Ouest africain ; il est situé dans une zone considérée comme car les conditions environnementales, surtout climatiques, ne sont pas très favorables à la production de dattes de bonne qualité [1]. La culture du palmier dattier au Mali est localisée dans les régions de Kayes et de l'Adrar des Ifoghars. Cette espèce a été introduite dans la région du massif de l'Adrar des Ifoghars (nord-est du Mali) à partir du Tassili et du Hoggar en Algérie.

Pour cela, des spécialistes du palmier dattier de la région de Touat (ouest du Sahara algérien) ont aidé au développement de cette culture dans le nord-est du Mali, surtout autour de Tessalit (région de Kidal) devenu site primaire de culture du dattier dans le pays.

Aujourd'hui, de petites palmeraies sont implantées dans diverses régions du Mali (Gao, Tombouctou, Kidal et Kayes) et des pieds isolés sont observés sur l'ensemble de la zone aride et semi aride du pays [2].

Traditionnellement, les habitants de cette zone étaient des nomades qui pratiquaient l'élevage. Avec l'installation de la sécheresse et la dégradation des pâturages, ils ont commencé à s'intéresser à l'agriculture pour assurer leur survie. La culture du dattier est l'une des spéculations qui a alors suscité l'intérêt des populations locales du fait des conditions écologiques de la région. C'est une zone qui connaît également une instabilité politique, en raison des conditions socio-économiques difficiles vécues par les populations du nord du Mali. L'objectif de nos recherches a été de contribuer au développement de ces régions afin d'assurer une certaine stabilité à ses habitants.

Nos travaux se sont inscrits dans le cadre des projets de développement de la phoeniciculture au Nord du Mali. Ils font partie des premières recherches qui se sont intéressées aux ressources phytogénétiques du palmier dattier au Mali, alors que de nombreuses études de ce type avaient été déjà menées dans divers autres pays africains : Kearny (1906) en Tunisie [3], Brown (1924) et Peyron et al. (1990) en Égypte [4, 5], Chevalier (1930) en Mauritanie [6], Dowson (1961) en Lybie [7], Maatalah (1970) en Algérie [8] et Popenoe (1973) dans plusieurs pays du Maghreb et du Golfe [9].

Notre étude s'est appuyée sur l'inventaire du patrimoine phoenicicole et la caractérisation variétale de quelques cultivars dans la région de Kidal au Mali.

\section{Matériel et méthodes}

\subsection{Présentation des sites d'étude}

La région de Kidal est située au nord du Mali, elle est limitée au nord par l'Algérie, au sud par la région de Gao, à l'est par le Niger et à l'ouest par la région de Tombouctou (figure 1).

L'inventaire du patrimoine phoenicicole de la région s'est déroulé dans neuf sites : Tessalit (extrême nord de la commune de Kidal), Télabit (à $120 \mathrm{~km}$ de la ville de Kidal), Tanezrouft (à $85 \mathrm{~km}$ au nord de Kidal), Tenzaoutène (à $350 \mathrm{~km}$ au nord-est de Kidal et à $8 \mathrm{~km}$ de la ville algérienne de Tin Zaouatène, Kidal (localisation des palmeraies mises en place par l'administration coloniale), Aguel-Hoc (à $95 \mathrm{~km}$ au sud de Tessalit), Tassidjimet et Tagharabat (ces deux sites forment une seule entité située au sud de Tessalit, à $50 \mathrm{~km}$ d'Aguel-Hoc), N'Tibdoc (à $75 \mathrm{~km}$ au sud-est de Tessalit) (figure 1) [2]. 
D'autres sites ne contiennent que des pieds isolés en effectifs très réduits ; c'est le cas de Tefaïnake, où nous avons dénombré seulement 19 pieds.

\subsection{Méthodologie de travail}

La méthodologie que nous avons utilisée pour inventorier les palmiers-dattiers présents dans la région de Kidal a été basée sur des observations qui ont résulté de quatre mois d'enquêtes : de mai à septembre 1997. Celles-ci ont été réalisées en trois phases principales [10]

- une phase de repérage commencée six mois avant la récolte qui a permis une prise de contact avec les agriculteurs et la localisation des différents sites de la zone d'étude ;

- une phase d'échantillonnage des palmiers et des fruits ; pour chaque cultivar identifié, nous avons choisi trois pieds, sains, adultes et en conditions de culture régulières ; les pieds ont été identifiés avec l'aide de spécialistes du palmier dattier de la région d'échantillonnage ;

- une phase d'échantillonnage complémentaire réalisée au cours de la campagne qui a suivi la première phase de récolte.

Les enquêtes sur le patrimoine phoenicicole ont été réalisées en se basant sur une fiche regroupant des informations sur le propriétaire, le nombre de palmiers dont il s'occupe, le nombre de palmiers productifs, le sexe des pieds, les cultivars présents, le nombre de pieds recensés par cultivar, le nombre de palmiers issus de graines, l'âge des pieds et la période de maturation.

La caractérisation des cultivars a été faite essentiellement sur :

- les caractères végétatifs du pied (forme, circonférence et hauteur du stipe, nombre de palmes, dimensions d'une palme verte de la couronne externe, densité des pennes sur une distance d'1 $\mathrm{m}$ de la palme, nombre d'épines, ainsi que leur densité sur $50 \mathrm{~cm}$ ) ;

- l'inflorescence (nombre, dimensions) ;

- le fruit (forme, poids, dimensions, couleur, consistance) ;
- la graine (forme, dimensions, poids, sillon) [11-13]

Ces caractères ont été étudiés sur trois palmiers de chaque cultivar. Les pieds en question devaient être sains, adultes et se développant dans de bonnes conditions de culture, ou, tout au moins, dans des conditions de culture acceptables. Les mesures ont été réalisées en palmeraies et en laboratoire $[1,13]$.

La caractérisation a été limitée à dix-sept cultivars recensés dans la région de Tessalit, qui regroupe tous les cultivars identifiés dans la région du Kidal, afin de limiter la variabilité due aux conditions d'environnement.

Il est à noter que, même en cas de caractérisations biochimique ou génétique [1416], la caractérisation morphologique reste nécessaire car elle permet de confirmer leurs résultats.

Les résultats ont été analysés par Analyse Factorielle des Correspondances multiples (AFCm), avec le logiciel STATITCF. Cette méthode d'analyse statistique nous a permis de disposer de la représentation synthétique de vastes ensembles de valeurs numériques, sous forme de visualisation graphique sur des axes principaux [17].

\section{Résultats et discussion}

\subsection{Caractéristiques du patrimoine phoenicicole}

\subsubsection{Nombre de palmiers recensés}

Le nombre des palmiers dattiers recensés dans la région de Kidal a dépassé 4000 pieds ; parmi eux, plus de 1700 pieds sont des palmiers productifs (tableau I). Avec 39,05\% de l'effectif total, la région de Tessalit se révèle être celle qui héberge le plus de palmiers-dattiers. Cette localité située près de la frontière algéro-malienne est considérée comme une aire primaire pour la distribution du dattier au Mali, faite à partir de l'Algérie.

Le nombre de palmiers productifs est apparu très élevé, surtout à Tessalit 
Tableau I.

Nombre total de palmiers dattiers et nombre de pieds productifs recensés dans la région de Kidal, au nord-est du Mali, au cours d'une prospection destinée à évaluer le patrimoine phoenicicole de cette région.

\begin{tabular}{lcc}
\hline Sites & Nombre total de pieds & Nombre de pieds productifs \\
\hline Aguel-Hoc & 90 & 10 \\
Kidal & 177 & 38 \\
N'Tibdoc & 98 & 38 \\
Tagharabat & 449 & 148 \\
Tanezrouft & 517 & 51 \\
Tassidjiment & 501 & 155 \\
Tefaïnake & 19 & 13 \\
Télabit & 568 & 258 \\
Tenzaouatène & 203 & 54 \\
Tessalit & 1680 & 992 \\
\hline Total région & 4302 & 1757
\end{tabular}

Tableau II.

Répartition des cultivars de palmiers dattiers dans trois sites de la région de Kidal au nord-est du Mali, tels qu'inventoriés au cours d'une prospection destinée à évaluer le patrimoine phoenicicole de cette région.

\begin{tabular}{lccc}
\hline Cultivar & \multicolumn{2}{c}{ Sites de la région de Kidal } \\
& Tessalit & Télabit & Tanezrouft \\
\hline Aboussekdi & Fréquent & N'existe pas & N'existe pas \\
Degla & Rare & Peu fréquent & N'existe pas \\
Gazoul El Bagra & Rare & N'existe pas & N'existe pas \\
Idamamane & Fréquent & N'existe pas & N'existe pas \\
Imoulaye & Rare & N'existe pas & N'existe pas \\
Lakamera & Peu fréquent & N'existe pas & N'existe pas \\
Oumakcha & Rare & N'existe pas & N'existe pas \\
Souboro El Satin & N'existe plus & N'existe pas & N'existe pas \\
Tadamant & Peu fréquent & Peu fréquent & N'existe pas \\
Takawel & Abondant & N'existe pas & N'existe pas \\
Tangalt & Abondant & N'existe pas & N'existe pas \\
Tassakh & Fréquent & N'existe pas & N'existe pas \\
Tergal & Fréquent & N'existe pas & N'existe pas \\
Tigaza & Rare & Abondant & Fréquent \\
Tilemsou & Peu fréquent & N'existe pas & N'existe pas \\
Tinacer & Rare & Peu fréquent & Rare \\
Tinelzizane & Rare & Fréquent & N'existe pas \\
Tinhadane & Peu fréquent & N'existe pas & N'existe pas \\
& &
\end{tabular}

(992 palmiers productifs, soit $59 \%$ de l'ensemble des palmiers productifs recensés) et à Télabit (258 palmiers, soit $45 \%$ de l'ensemble). Le taux de dattiers productifs se révèle très élevé à Tafaïnake du fait que le nombre de palmiers-dattiers recensés y est très réduit.

Le palmier dattier aurait été introduit à Télabit et à Tanezrouft à partir de Tessalit ; puis il l'aurait été à Tassidjimet et à Tagharabat, considérées alors comme palmeraies de troisième génération.

\subsubsection{Cultivars de palmiers dattiers}

La diversité des palmiers-dattiers a été étudiée dans les trois sites les plus pourvus de la région : Tessalit, Télabit et Tanezrouft (tableau II). Le site de Tessalit semble être le seul site qui contienne les 18 cultivars recensés, mais le nombre de leurs représentants y est très différent. À noter que le cultivar Souboro El Satin, qui avait été recensé durant les premières enquêtes, n'a pas été retrouvé sur le terrain. La présence de l'ensemble des cultivars à Tessalit montre que la culture du palmier dattier y est bien installée. Tel n'est pas le cas dans les deux autres sites puisque seuls cinq cultivars ont été observés à Télabit (Degla, Tadamant, Tigaza, Tinacer et Tinelzizane) et deux à Tanezrouft (Tigaza et Tinacer).

Plus de $70 \%$ de ces cultivars que nous avons recensés sont des palmiers à dattes demi-sèches ou demi-molles. Les cultivars à dattes molles ne sont pas très cultivés dans la région de Kidal du fait de la difficulté de conservation de leurs fruits et des conditions climatiques de la zone.

\subsubsection{Nombre de palmiers-dattiers par cultivar}

Dans les localités échantillonnées, nos enquêtes ont permis de recenser plus de 4300 palmiers-dattiers dont 1070 pieds appartenaient à des cultivars. Les autres plants correspondent à des pieds sans identification, qui seraient des palmiers issus de graines, appelés "Dguels " ou " Dgouls ".

Bien que le nombre de palmiers par cultivar soit variable, il semble que les cultivars Aboussekdi, Takawel, Tangalt, Tergale et Tigaza soient les cultivars dominants de la région. Les cultivars Gazoul El Bagara, Oumakcha, Tinacer et Tinelzizane n'ont été recensés qu'en très petit nombre. 
À coté de ces cultivars, qui représentent $24,84 \%$ des palmiers recensés, il existe un nombre très élevé de plants issus de graines (75,16\% de l'effectif total). Ce pourcentage dépasse celui qui avait été enregistré par Saaidi au Maroc (50 \% de l'effectif total) [18]. Le grand nombre de plants obtenus à partir de graines dans la région de Kidal illustrerait le caractère traditionnel de la culture du dattier. En effet, seule la multiplication végétative (par rejets) permet de maintenir les caractéristiques plus ou moins stables des cultivars sélectionnés [1].

\subsubsection{Période de maturation des cultivars}

La majorité des cultivars étudiés dans la région de Kidal ont une production précoce. En effet, $61,29 \%$ des pieds recensés produisent des dattes qui arrivent à maturité en juin et juillet. Les autres pieds (38,71\%), considérés comme tardifs, se récoltent en aout et septembre. Ils sont souvent exposés au risque des pluies. En Algérie, la période de maturation des dattes s'étale entre juin et décembre, période relativement longue par rapport à celle enregistrée au nord du Mali. Les variations de température sont responsables de ces différences de périodes de maturation. Ce sont elles également qui définissent les aires de répartition des différents cultivars [19].

\subsubsection{Effectif des palmiers mâles}

L'abandon des palmeraies qui se développent alors naturellement et la culture par semis de graines pourraient être à l'origine du recensement d'un nombre important de pieds mâles ou "Dokkars". En effet, dans les palmeraies spontanées, le nombre de pieds mâles est sensiblement égal à celui des pieds femelles [1], alors qu'en culture industrielle, la proportion des pieds mâles n'est que de $2 \%$ [20]. Aujourd'hui, une proportion de $4 \%$ de pieds mâles serait préconisée dans de telles cultures [21].

Dans de nombreuses localités échantillonnées, le nombre de palmiers mâles s'est révélé très proche de celui des femelles. Dans certaines autres, il est même apparu supérieur à celui des pieds femelles (cas de Télabit, Tagharabat, Tessalit).
Tableau III.

Structure d'âge des palmiers dattiers de la région de Kidal au nordest du Mali (\%), tels qu'inventoriés au cours d'une prospection destinée à évaluer le patrimoine phoenicicole de cette région.

\begin{tabular}{lccc} 
Site de la région de Kidal & \multicolumn{3}{c}{ Âge des palmiers dattiers } \\
\cline { 2 - 4 } & $<10$ ans & 10 ans à 50 ans & $>50$ ans \\
Aguel-Hoc & 88,89 & 11,11 & - \\
Kidal & 57,06 & 10,17 & 32,77 \\
N'Tibdoc & 59,18 & 38,78 & 2,04 \\
Tagharabat & 30,96 & 69,04 & - \\
Tanezrouft & 78,53 & 15,09 & 06,38 \\
Tassidjiment & 56,69 & 43,31 & - \\
Tefaïnake & - & 100,00 & - \\
Télabit & 1,23 & 73,77 & 25,00 \\
Tenzaouatène & 60,10 & 39,90 & - \\
Tessalit & 9,00 & 80,00 & 11,00
\end{tabular}

Dans le site d'Aguel-Hoc, l'effectif des mâles est relativement faible $(8,88 \%)$, alors qu'à Tanezrouft et Tenzaouatène, nous avons recensé, respectivement, $12,37 \%$ et 13,79 \% pieds mâles.

\subsubsection{Effectif des palmiers dattiers issus de graines (Dgouls)}

Le site de Tessalit présente un faible pourcentage de palmiers dattiers issus de graines puisque seuls $8,45 \%$ de l'effectif total des pieds de ce site sont de cette origine. Dans ce site très ancien, la sélection des pieds et la propagation végétative sont très pratiquées.

Le site de Télabit n'héberge que 1,25\% " Dgouls " ; mais sa palmeraie se caractérise par un nombre très élevé de pieds mâles.

Dans les autres sites échantillonnés, les effectifs de "Dgouls" sont très élevés puisque leurs taux varient entre 30,95\% et $81,11 \%$. Cela illustrerait l'aspect traditionnel de la phoeniciculture dans la région de Kidal.

\subsubsection{Age des palmiers dattiers}

Le nombre des pieds jeunes semble être très élevé dans les sites de Aguel-Hoc, Kidal, N'Tibdoc, Tanezrouft, Tassidjiment et Tenzaouatène puisqu'ils dépassent $50 \%$ dans ces palmeraies (tableau III). Cette observation pourrait être liée à la sédentarisation 


\section{S. Babahani et al.}

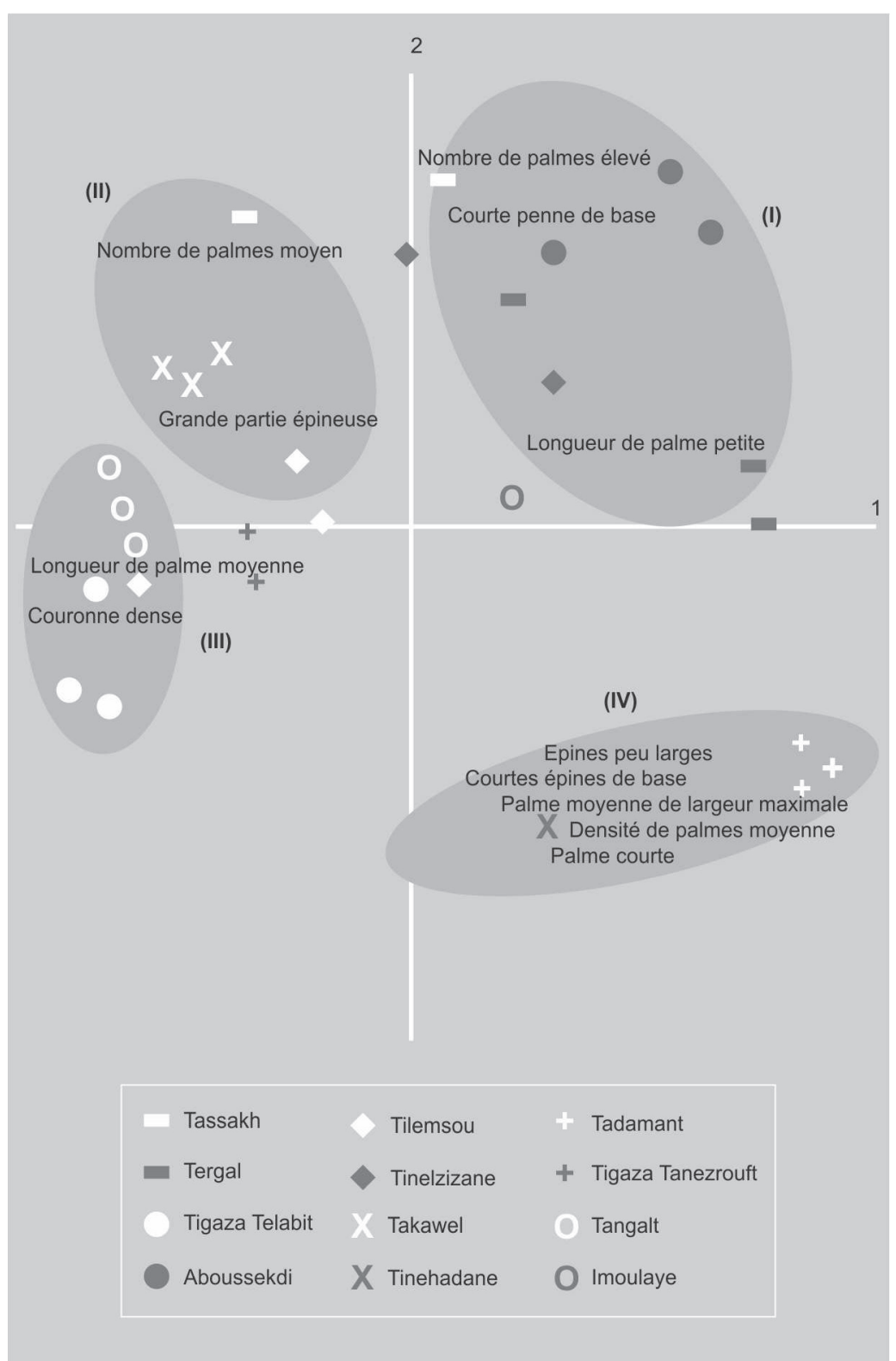

Figure 2.

Représentation des deux premiers axes d'une analyse factorielle des correspondances multiples appliquée à certains caractères végétatifs de 12 cultivars de palmiers dattiers répertoriés dans la région de Kidal au nordest du Mali (représentation de trois pieds par cultivar). des pasteurs nomades, due au fait de la perte de leurs bétails à cause de sécheresses répétées [2].

\section{2. Étude de la variabilité des cultivars de palmiers dattiers}

\subsubsection{AFCm sur les caractères végétatifs des cultivars}

L'analyse factorielle des correspondances multiples menée sur les caractères végétatifs des cultivars de palmiers dattiers échantillonnés a permis de discriminer quatre groupes d'individus (figure 2).

La variabilité intra-cultivar s'est révélée plutôt faible pour les individus des cultivars Aboussekdi, Tadamant, Takawel et Tigaza Télabit. Cette variabilité a été plus importante chez les autres cultivars.

La distinction entre les cultivars à partir des caractères végétatifs se révèle difficile [1]. En effet, les groupes n'apparaissent pas vraiment homogènes quant aux cultivars qu'ils contiennent.

Les individus du cultivar Tigaza Télabit semblent former un groupe différentié par rapport à ceux du cultivar Tigaza Tanezrouft, qui parait plus difficile à discriminer. Ces individus sont caractérisés principalement par une longueur de palme moyenne [22] et une couronne dense.

\subsubsection{AFCm sur les caractères des inflorescences}

L'analyse factorielle des correspondances multiples menée sur les caractères des inflorescences individualise également quatre groupes d'individus. Comme pour les caractères végétatifs, les caractéristiques des inflorescences permettent de regrouper les individus de certains cultivars (Tadamant, Takawel, Tangalt et Tilemsou) (figure 3). L'interprétation des axes montre que le cultivar Tadamant se caractériserait essentiellement par une faible partie sans fruits des branchettes du milieu du régime ; les cultivars Tangalt et Tilemesou auraient un nombre élevé de fruits au sommet du régime. Les autres cultivars présentent une certaine variabilité intra-cultivar.

Les individus du cultivar Tigaza Tanezrouft semblent être mieux discriminés par les caractères des inflorescences que par les caractères végétatifs. Ils se distinguent surtout par une partie ramifiée moyenne.

Les caractéristiques de l'inflorescence mettent en évidence une importante variabilité inter-cultivars. Toutefois, pour ces caractères, il semble qu'il existe une proximité phénotypique entre les deux cultivars Tilemsou et Tangalt. Globalement, les caractères des inflorescences des dattiers à Kidal 
paraissent moins marqués que ceux rapportés par Nixon et Carpenter [23].

\subsubsection{AFCm sur les caractères des fruits et des graines}

L'analyse factorielle des correspondances multiples menée sur les caractéristiques des fruits et des graines a mis en évidence trois groupes d'individus (figure 4 ). Ces caractères discriminent nettement les individus des cultivars Takawel, Tilemsou et même ceux du cultivar Tergal. Ce résultat confirme ceux de Nixon [22].

Les individus du cultivar Aboussekdi et Tilemsou sont caractérisés par un épicarpe lisse du fruit. Ceux du cultivar Tilemsou présenteraient en outre des fruits à consistance molle. Les individus du cultivar Takawel sont caractérisés par un fruit de forme droite et qui, au stade Tmar (stade de maturité complète), serait de couleur ombrée.

\subsubsection{AFCm globale sur les caractères discriminants}

L'analyse globale des caractères les plus discriminants a permis de mettre en évidence trois groupes distincts d'individus. La variabilité entre cultivars est révélée surtout par la prise en compte des caractères de l'inflorescence et des fruits et moins par celle des caractères végétatifs (figure 5). Cette observation confirme les résultats obtenus par Hannachi et al. [11] et Belguedj [10]. Notre analyse met en évidence une proximité entre les individus des cultivars Tigaza Télabit et Tigaza Tanezrouft.

Les individus des cultivars Degla, Gazoul El Bagra, Lakamera, Oumakcha et Tinacer n’ont pu être discriminés par les caractères étudiés. Il s'agit pour la plupart de cultivars rares (tableau II) dont la sélection serait relativement faible par rapport aux cultivars mieux représentés.

En revanche, l'ensemble des caractères considérés discrimineraient, au sein du cultivar Tigaza également considéré comme rare, deux " clones " différents : Tigaza Télabit et Tigaza Tanezrouft, tous deux classés dans un même groupe de l'AFCm globale. Ce cultivar est très connu au sud-ouest algérien.

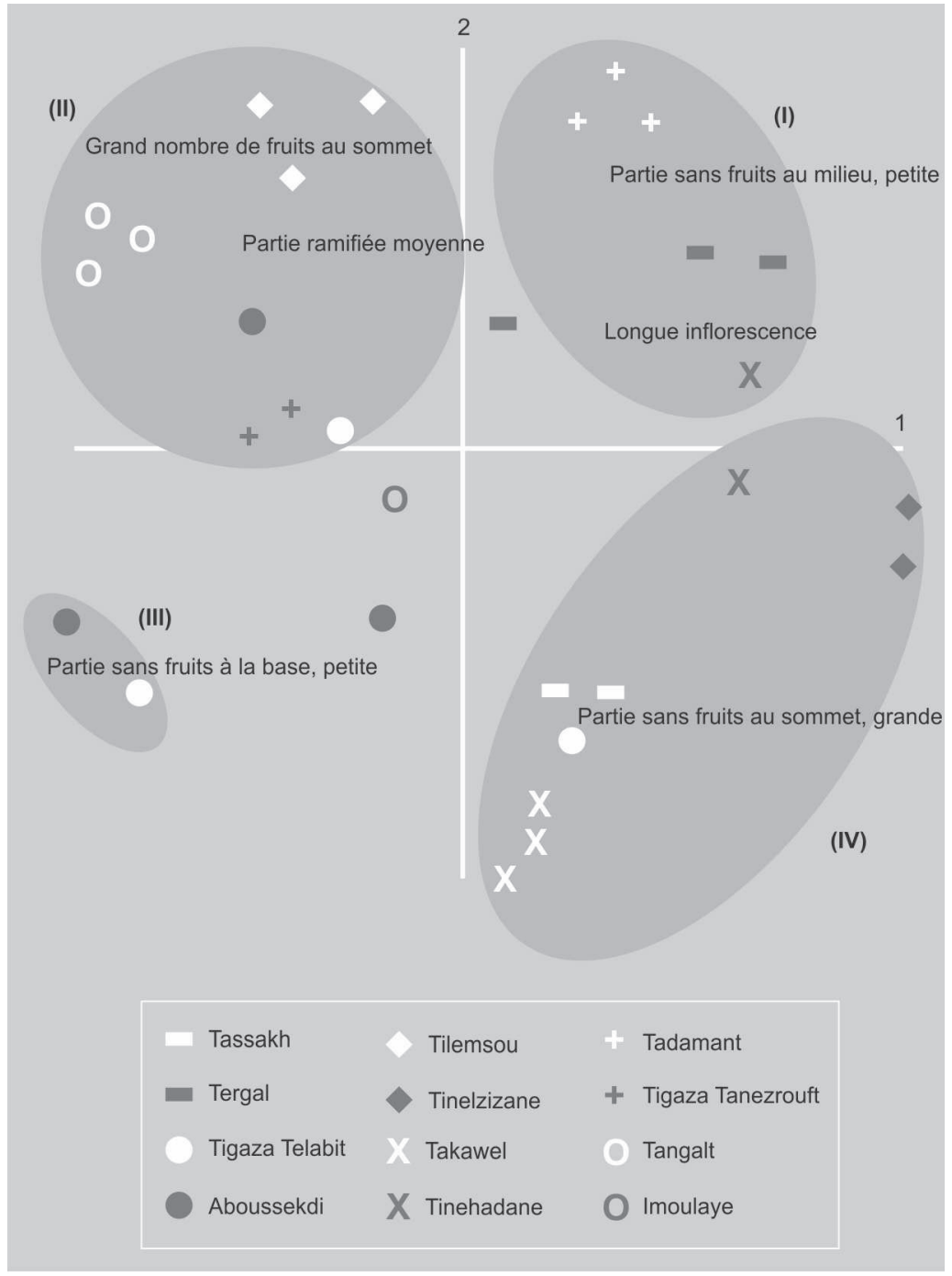

Une étude comparative des caractères de couleur du fruit, d'aspect de l'épicarpe, de digestibilité et de période de maturation a montré qu'il existait une similitude entre les individus de Tigaza Télabit et Tigaza Tanezrouft, originaires d'Algérie, et les palmiers Tigaza de Tidikelt en Algérie [11].

\section{Conclusion}

Figure 3.

Représentation des deux premiers axes d'une analyse factorielle des correspondances multiples appliquée à certains caractères des inflorescences de 12 cultivars de palmiers dattiers répertoriés dans la région de Kidal au nord-est du Mali (représentation de trois pieds par cultivar).

Les prospections dans la région de Kidal, au nord-est du Mali, ont permis de recenser plus de 4000 palmiers dattiers dont plus de 1700 pieds productifs. 


\section{S. Babahani et al.}

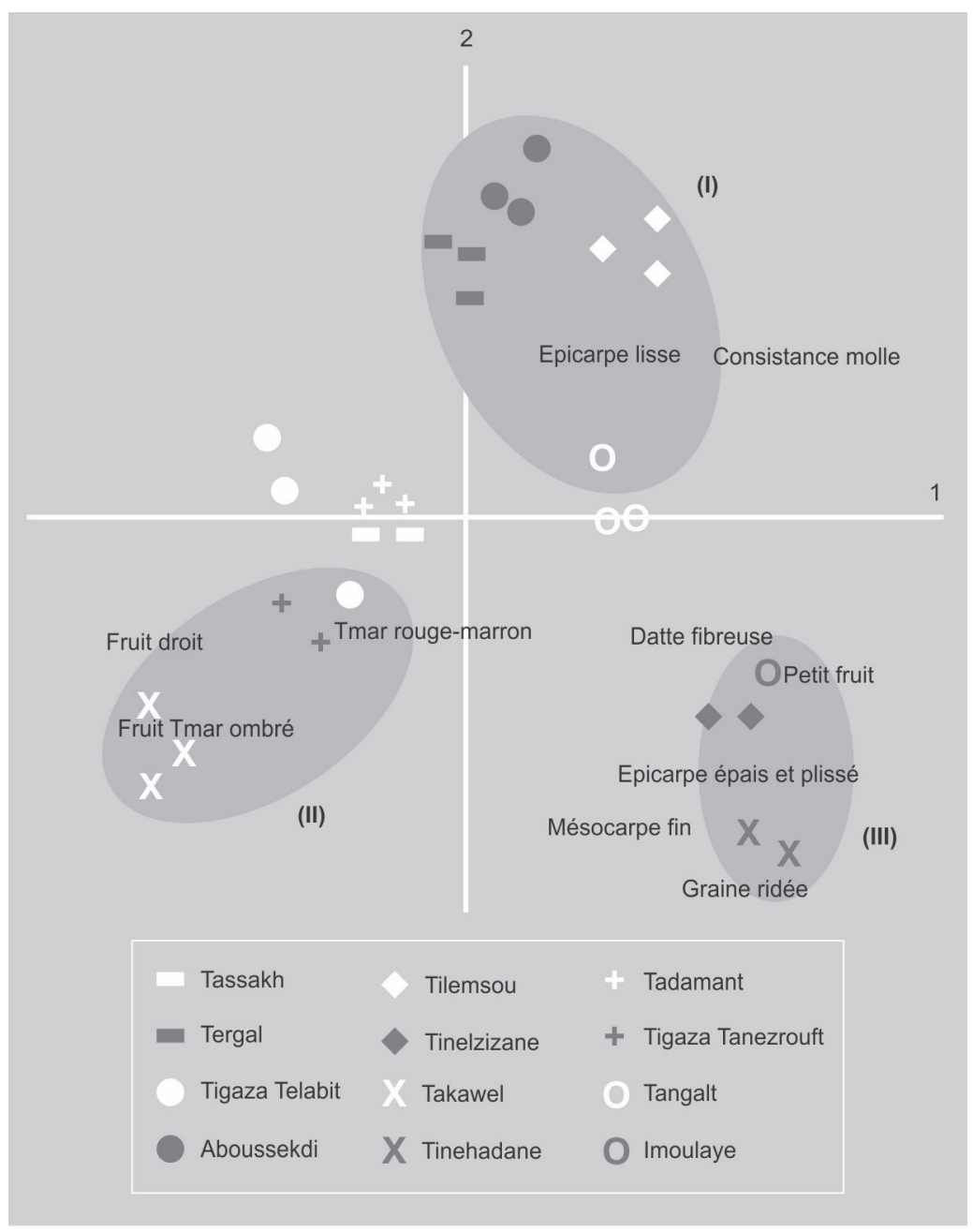

Figure 4.

Représentation des deux premiers axes d'une analyse factorielle des correspondances multiples appliquée à certains caractères des fruits et des graines de 12 cultivars de palmiers dattiers répertoriés dans la région de Kidal au nord-est du Mali (représentation de trois pieds par cultivar). révèlerait qu'il s'est fait une forte sélection des pieds d'un même cultivar et que les cultivars introduits principalement de l'Algérie se seraient bien adaptés aux conditions écologiques locales du nord du Mali. Des proximités ont été mises en évidence entre deux cultivars de Tigazas locaux et la Tigaza de l'Algérie.

La sensibilisation des populations de cette région sur la nécessité de développer le patrimoine phoenicicole et de préserver sa diversité doit être encouragée afin de préserver la phoeniciculture à Kidal, considéré comme région instable.

\section{Références}

[1] Munier P., Le palmier dattier, G.-P. Maisonneuve et Larose, Paris, France, 1973.

[2] Babahani S., Hannachi S., Togo A., Existe-til une phoeniciculture au Mali ? Cas de la région de Kidal, Cah. Agric. 19 (3) (2010) 227-230.

[3] Kearny T.H., Date varieties and date culture in Tunis, U.S. Bur. Plant Ind. Bul. 92 (1906) 112.

[4] Brown T.W., Date palm in Egypt, Tech. Sci. bull. Minist. Agric., Hortic. Sect. 43, pp. 1115, Cairo, Egypt, 1924.

[5] Peyron G., Gay F., Rafat A.A., Contribution à l'étude du patrimoine génétique phoenicicole en Égypte, Options Méditerr. Ser. A 11 (1990).

[6] Chevalier A., Le dattier en Mauritanie, Rev. Bot. Appl. 10 (1930) 372-376.

[7] Dowson V.H.W., Report to the Government of Libya on date production, FAO / EPTA, Rep. $n^{\circ}$ 1263, Rome, Italy, 1961.

[8] Maatalah S., Contribution à la valorisation de la datte algérienne, INA, Mém., El Harrach, Alger, Algérie, 1970.

[9] Popenoe P., The date palm, Field Res. Proj., Coconut Grove, Miami, U.S.A., 1973.

[10] Belguedj M., Les ressources génétiques du palmier dattier: Caractéristiques des cultivars de dattiers dans les palmeraies du sudest algérien, INRAA, Alger, Algérie, 2002.

[11] Hannachi S., Khitri D., Ben Khalifa A., Brac de la Perrière R.A., Inventaire variétal de la palmeraie algérienne, ANEP, Rouiba, Alger, Algérie, 1998. 
[12] Rhouma A., Le palmier dattier en Tunisie. I Le patrimoine génétique, Vol. 1, Arabesques Ed. \& Créat., Tunis, Tunisie, 1994.

[13] Anon., Descripteurs du palmier dattier (Phoenix dactylifera L.), Inst. Int. Ressour. Phytogénét. (IPGRI), IPGRI / INRA, Tunis, Tunisie, 2005.

[14] Ould Mohamed S., Rhouma S., Zehdi S., Marrakchi M., Trifi M., Molecular characterization of Mauritanian date palm cultivars using plasmid-like DNAs markers, Biol. Plant. 51 (2007) 169-172.

[15] Elshibli S., Korpelainen H., Microsatellite markers reveal high genetic diversity in date palm (Phoenix dactylifera L.) germplasm from Sudan, Genetica 134 (2008) 251-260.

[16] Ouafi S., Bounaga N., Lebreton Ph., Bouguedoura N., Contribution à l'étude des hétérosides flavoniques du palmier dattier. Recherche de marqueurs des cultivars algériens, Rev. Rég. Arid. 2 (2008) 379-385.

[17] Lebart L., Morineau A., Piron M., Statistique exploratoire multidimensionnelle, $2 \mathrm{e}$ ed., Dunod, Paris, France, 1995.

[18] Saaidi M., Amélioration génétique du palmier dattier au Maroc, critères de sélection, technique et résultats, Options Méditerr. Sér. A 11 (1990).

[19] Berbendi A.R., Les palmiers, techniques et perspectives, ACSAD, Damas, Syrie, 2000 (en arabe).

[20] Nixon R.W., Growing dates in the United States, USDA, Agric. Inf. Bull. No 207, U.S.A., 1966.

[21] Hussein F., Pollinisation du dattier et son effet sur la production et la qualité des fruits, in: Symp. Palmier dattier, Univ. Roi Faysal, El Hassa, Arabie Saoudite, 1983, pp.15-24 (en arabe).

[22] Nixon R.W., Date culture in French North Africa and Spain, Date Growers' Inst. Rep. 27 (1950) 15-21.

[23] Nixon R.W., Carpenter J.B., Growing dates in the United States, Agric. Inf. Bull. 207 (1978).

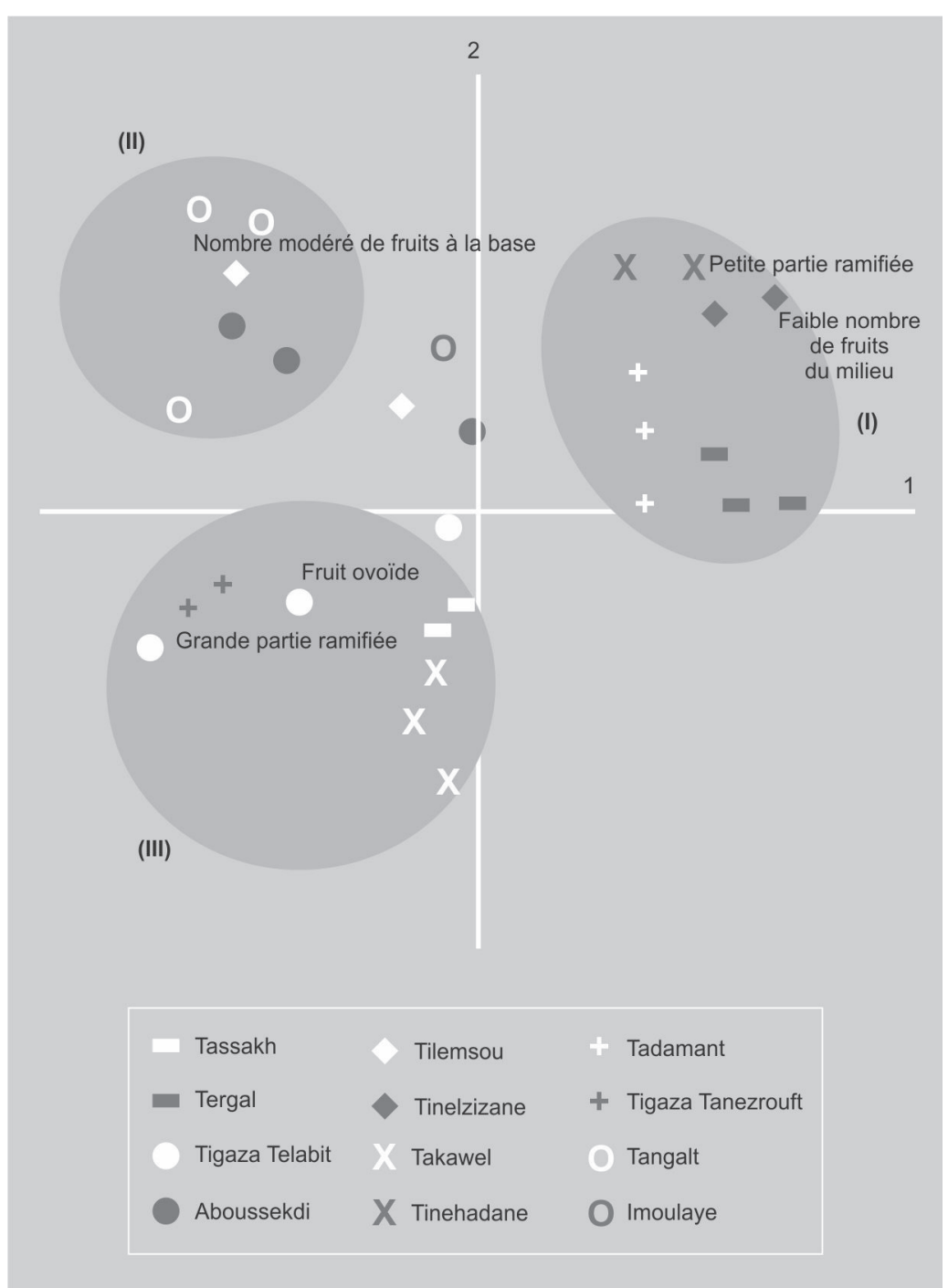

\section{Figure 5.}

Représentation des deux premiers axes d'une analyse factorielle des correspondances multiples globale prenant en compte l'ensemble des caractères végétatifs et des caractères morphologiques d'inflorescences, de fruits et de graines, utilisés pour étudier 12 cultivars répertoriés dans la région de Kidal au nordest du Mali (représentation de trois pieds par cultivar). 


\section{S. Babahani et al.}

\section{Estudio del patrimonio datilero de Kidal, al norte de Mali.}

Resumen - Introducción. Las poblaciones de Kidal, al norte de Mali, tradicionalmente nómadas, se orientan progresivamente hacia la agricultura, por causa de las recurrentes sequías. El cultivo de la palmera datilera aún sigue poco elaborado, a pesar de estar presente en esta región. Nuestro estudio se inscribió en el marco de proyectos de desarrollo de la fenicicultura al norte de Mali. Su objetivo fue el de inventariar y caracterizar los recursos fitogénicos de la palmera datilera, de modo a poder proyectar estrategias de desarrollo de dicho patrimonio. Material y métodos. Nuestro estudio se llevó a cabo en la región de Kidal, en nueve parcelas principales, conocidas desde hace tiempo por el cultivo de la palmera datilera. Determinamos el número total de pies observados en la región, la efectividad de los pies productivos, el reparto de sexos, los cultivares representados, la edad de las cepas y sus periodos de maduración. Se estableció una característica común para los principales cultivares, basada en los caracteres vegetativos y morfológicos de las inflorescencias, en los frutos y en las semillas. Resultados y discusión. Inventariamos más de 4000 palmeras datileras en la región muestreada. Las proporciones de las palmeras macho resultaron ser elevadas. La parcela de Tessalit parece albergar el palmeral más importante y antiguo. Representa el 39\% de la efectividad total de las palmeras datileras inventariadas. Nuestro estudio permitió identificar 18 cultivares. Los análisis factoriales de las correspondencias múltiples sobre la caracterización de los cultivares de la región de Kidal mostraron que la variabilidad entre unos y otros cultivares parece ser muy importante, independientemente de los caracteres estudiados. Por lo contrario, la variabilidad dentro de los propios cultivares es floja. Se definieron proximidades entre los cultivares de Tigazas locales y la Tigaza argelina.

Mali / Pboenix dactylifera / recursos genéticos / encuestas / características del rodal / variación genética 